\title{
Simultaneous, Simple and Rapid Determination of Five Bioactive Free Anthraquinones in Radix et Rhizoma Rhei by Quantitative ${ }^{1} \mathrm{H}$ NMR
}

\author{
Jian-Wei Dong, Le Cai, *Yun-Shan Fang, Wei-He Duan, Zhen-Jie Li and Zhong-Tao Ding* \\ Key Laboratory of Medicinal Chemistry for Natural Resource, Ministry of Education, School of \\ Chemical Science and Technology, Yunnan University, 650091 Kunming, P. R. China
}

\begin{abstract}
Radix et Rhizoma Rhei has been recognized for centuries in traditional medicine for its multiple pharmacological actions. The free anthraquinones including physcion, chrysophanol, emodin, rhein, and aloe-emodin are the main bioactive components in Radix et Rhizoma Rhei. In the present study, a fast quantitative ${ }^{1} \mathrm{H}$ nuclear magnetic resonance (q-HNMR) method for the determination and quantitation of five free anthraquinones in Radix et Rhizoma Rhei was developed. Validation of the quantitative method was performed in terms of specificity, accuracy, precision, and stability. The results showed that the solvent acetone- $d_{6}$ enabled satisfactory separation of the signals to be integrated. Five anthraquinones in Radix et Rhizoma Rhei could be quantified accurately using featured signals from ${ }^{1} \mathrm{H}$ NMR. This work implied that q-HNMR represents a feasible alternative to high-performance liquid chromatography (HPLC)-based methods for quantitation of anthraquinones in Radix et Rhizoma Rhei and is suitable for the quality control of Radix et Rhizoma Rhei.
\end{abstract}

Keywords: Radix et Rhizoma Rhei, anthraquinone, quantitative ${ }^{1} \mathrm{H}$ NMR, validation, quality control

\section{Introduction}

Radix et Rhizoma Rhei is the rhizome or root of perennial herbaceous plants Rheum palmatum L., Rheum officinale Baill, or Rheum tanguticum Maxim. ex Balf, ${ }^{1}$ which are not only used as healthy food in many countries including China, but also represent one of the most popular medicinal herbs used in clinical practice. ${ }^{2}$ Radix et Rhizoma Rhei has been used as a traditional medicine for centuries on account of its pharmacological effects (antipyretic, antineoplastic, antipasmolytic, antibacterial, laxative, hemostatic, and antispasmodic). ${ }^{3,4}$ Previous research revealed that the components of Radix et Rhizoma Rhei contain anthraquinones, stilbenes, dianthrones, anthocyanins, flavonoids, galloyglucoses, phenylbutanones, organic acids, etc. ${ }^{5}$ Among them, five free anthraquinones (physcion, chrysophanol, emodin, rhein, and aloe-emodin) listed in Figure 1 are the main effective constituents. ${ }^{1,6-8}$

Herbal medicines (HMs) or traditional Chinese medicines (TCMs) have been widely used for treating various diseases for thousands of years in most oriental countries. It is urgently necessary to establish the feasible and effective

*e-mail: ztding@ynu.edu.cn, caile@ynu.edu.cn

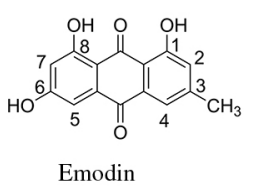

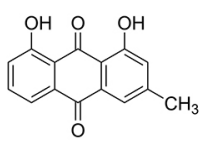

Chrysophanol

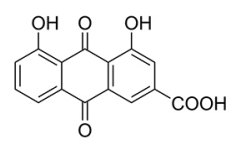

Rhein

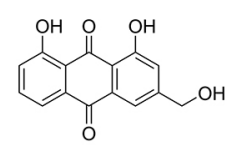

Aloe-emodin

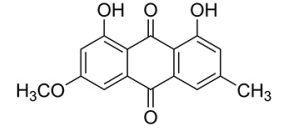

Physcion
Figure 1. Free anthraquinones of Radix et Rhizoma Rhei.

quality control pattern for HMs or TCMs to ensure their reliability for pharmacological and clinical utilization. ${ }^{9}$ Therefore, the analysis of the main bioactive components in HMs or TCMs is very important for quality control of crude drugs and elucidating the therapeutic action of HMs or TCMs. ${ }^{10}$ High-performance liquid chromatography (HPLC) for determination of five free anthraquinones is frequently used for quality control of Radix et Rhizoma Rhei according to the Chinese Pharmacopoeia and related literatures. ${ }^{1,9,11-19}$ Moreover, thin-layer chromatography (TLC), ${ }^{20,21}$ capillary electrophoresis (CE), ${ }^{22,23}$ ultra-performance liquid chromatography (UPLC), ${ }^{6,24}$ UPLC-mass spectrometry (MS), ${ }^{3}$ and high speed counter current chromatography 
$(\mathrm{HSCCC})^{25}$ also have been reported for determination of the main bioactive components in Radix et Rhizoma Rhei. However, all the analytical methods above for determining and quantifying Radix et Rhizoma Rhei suffer from the intrinsic limitations of chromatography-based methods, namely, the need of standard compounds for analyte identification or quantification and the relatively long analysis times. In addition, low reproducibility frequently occurred, which might be caused by column performance worsening over time, retention time drifts and insufficient separation. ${ }^{26}$

Quantitative nuclear magnetic resonance (q-NMR) is a fast and accurate method for the determination and quantitation of natural products ${ }^{27,28}$ including natural compounds, ${ }^{29}$ plant extracts, ${ }^{26,30,31}$ drugs, ${ }^{32,33}$ animal extracts, ${ }^{34}$ blood, ${ }^{35-37}$ food,${ }^{38}$ drink, ${ }^{39}$ etc. de Graaf et al.${ }^{34}$ reported the quantitation of amino acids from rat brain extracts by high-resolution NMR spectra. Neumüller et al. ${ }^{40}$ developed a fast and robust method to determine phenoyl and acetyl esters of polysaccharides by quantitative ${ }^{1} \mathrm{H}$ NMR (q-HNMR). Valverde and This ${ }^{41}$ revealed that ${ }^{1} \mathrm{H}$ NMR is applicable for the determination and quantitation of photosynthetic pigments from green bean (Phaseolus vulgaris L.) in a fast analytical way, which provides more information on chlorophyll derivatives (allomers and epimers) than UV-Vis spectroscopy. It's worth noting that traditional NMR and q-NMR are accepted by the International Conference on Harmonization (ICH). ${ }^{42}$ Malz and Jancke ${ }^{43}$ demonstrated that q-NMR is robust for quantitative instrument change. Sharma et al. ${ }^{44}$ defined a protocol for q-NMR applications with parental drugs. Thus, q-NMR is an effective quantitative method for rapid potency analysis compared with other determination and quantitation. ${ }^{45}$

In the present study, a fast method for determination and quantitation of five bioactive free anthraquinones in Radix et Rhizoma Rhei by q-HNMR was developed. The present study suggested that q-HNMR represents a feasible alternative to HPLC-based methods for the quantitation of anthraquinones in Radix et Rhizoma Rhei and is suitable for the quality control of Radix et Rhizoma Rhei.

\section{Experimental}

\section{Chemicals}

Emodin ( $\geq 98 \%)$, aloe-emodin $(\geq 98 \%)$, and chrysophanol ( $\geq 99.6 \%)$ were obtained from the National Institute for the Control of Pharmaceutical and Biological Products (Beijing, China). Rhein $(\geq 98 \%)$, physcion (>98\%), and 1,4-dioxane ( $\geq 99.0 \%)$ were purchased from
Aladdin Chemistry Co. Ltd. (Shanghai, China). Acetone- $d_{6}$ (99.9 atom\% $d$, contains $0.03 \%(\mathrm{v} / \mathrm{v})$ tetramethylsilane (TMS)), chloroform- $d$ (99.8 atom\% $d$, contains $0.03 \%(\mathrm{v} / \mathrm{v})$ TMS), and dimethyl sulfoxide (DMSO)- $d_{6}$ ( 99.9 atom $\% d$, contains $0.03 \%$ (v/v) TMS) were obtained from SigmaAldrich Co. LLC (St. Louis, MO, USA). Methanol (HPLC grade) was purchased from Honeywell Burdick \& Jackson Research Chemicals (Ulsan, Korea). The water (resistivity $\geq 18.25 \mathrm{M} \Omega \mathrm{cm}^{-1}$ ) used was purified with a purity water system (Chengdu, China). All other chemicals used were of analytical grade.

\section{Sample preparation}

Rheum palmatum L. and Rheum officinale Baill were obtained from Chinese herbal medicine market in Kunming, Yunnan Province, China, in November 2013, and were identified by Prof Shugang Lu from School of Life Sciences, Yunnan University. Two voucher specimens [2013-dh-01 (Rheum palmatum L.) and 2013-dh-02(Rheum officinale Baill)] have been deposited in the Key Laboratory of Medicinal Chemistry for Natural Resource, Ministry of Education, Kunming, Yunnan, China.

Two Radix et Rhizoma Rhei (Rheum palmatum and Rheum officinale) were ground to 60 mesh. Two powders (5.000 g each) were immersed in $50 \mathrm{~mL}$ chloroform for $24 \mathrm{~h}$, respectively, and ultrasonic extracted for three times (30 min each). The extracting solution was decanted, filtered under vacuum at $\leq 40{ }^{\circ} \mathrm{C}$, and concentrated in a rotary evaporator. Two crude extracts were thus obtained as Rheum palmatum L. (RP, $80.1 \pm 3.5 \mathrm{mg}$ ) and Rheum officinale Baill (RO, $82.8 \pm 2.9 \mathrm{mg}$ ) extracts, respectively. The extracts ( $2 \mathrm{mg}$, each) dissolved in deuterated solvents $(400 \mu \mathrm{L})$ containing $0.005 \%(\mathrm{v} / \mathrm{v})$ 1,4-dioxane [internal standard (IS)] were used for ${ }^{1} \mathrm{H}$ NMR assay.

\section{${ }^{1} \mathrm{H}$ NMR measurements}

All NMR spectra were recorded on a Bruker Avance $400 \mathrm{MHz}$ NMR spectrometer (Bruker-Biospin GmbH, Germany) with the $5 \mathrm{~mm}$ NMR tube. All samples were dissolved in acetone- $d_{6}$ or other deuterated solvents at the same concentration $\left(5.0 \mathrm{mg} \mathrm{mL}^{-1}\right) .{ }^{1} \mathrm{H}$ NMR spectra were acquired using the Bruker zg30 pulse program with the following settings: relaxation delay (D1) $=5 \mathrm{~s}$, size of free induction decay $(\mathrm{FID})(\mathrm{TD})=65536$, number of scans $(\mathrm{NS})=32$, spectral width $(\mathrm{SW})=16.4990 \mathrm{ppm}$, acquisition time $(\mathrm{AQ})=4.96 \mathrm{~s}$, requested probe temperature $(\mathrm{TE})=298.0 \mathrm{~K}$. All data acquisition and processing were done with Bruker Topspin 3.1. Signals of five free anthraquinones were determined according to the standards and quantitation 
according to the integral referred to the 1,4-dioxane from the ${ }^{1} \mathrm{H}$ NMR spectroscopy.

\section{q-HNMR method validation}

All samples were used for determination of precision, stability, and accuracy. For precision assay, $10.0 \mathrm{mg}$ of sample were accurately weighed and dissolved in $2000 \mu \mathrm{L}$ acetone, and then $400 \mu \mathrm{L}$ of mixture was transferred to the NMR tube. After the acetone was removed under vacuum, samples ( $2 \mathrm{mg}$ ) were re-dissolved in $400 \mu \mathrm{L}$ of the NMR solvent. Samples were stored in the dark at $4{ }^{\circ} \mathrm{C}$ until analysis, which was always determined within $12 \mathrm{~h}$ from the preparation of the solution. For stability assay, all samples were investigated in five consecutive days. Each day, three replicates were prepared and analyzed three times each. Repeatability was determined by comparing results from multiple spectra acquisition of the same sample. Results of precision, stability, and repeatability experiments were expressed as relative standard deviation (RSD, \%).

HPLC analysis was used to evaluate accuracy of q-HNMR method. The accuracy was expressed as RSD between the results obtained from q-HNMR and HPLC. The determination and quantitation of five anthraquinones in Radix et Rhizoma Rhei were measured according to a validated method. ${ }^{19}$ All samples and standards were filtered through a $0.45 \mu \mathrm{m}$ filter before injection into an Agilent 1260 Infinity Series system equipped with an Agilent G1315D diode array detector (DAD), Agilent G1311C quaternary pump, Agilent Zorbax SB-C18 $(250 \times 4.6 \mathrm{~mm}$ i.d., $5 \mu \mathrm{m}$ ) column (SN: USCL054040), and an OpenLAB CDS Chemstation Edition (version C.01.05 [35]) data processing station. An elution system consisting of solvent A $(0.1 \%$ phosphoric acid)-solvent B (methanol) $(15: 85$, $\mathrm{v} / \mathrm{v}$ ) was used for analysis. The peaks were confirmed by the UV absorptions at the wavelength of $254 \mathrm{~nm}$ and the retention times. The flow rate was $1.0 \mathrm{~mL} \mathrm{~min}^{-1}$, the column temperature was set at $35^{\circ} \mathrm{C}$, and the injection volume was $10 \mu \mathrm{L}$. All samples and standards were accurately weighed and dissolved in acetone to obtain a final concentration of 1.0 and $0.20 \mathrm{mg} \mathrm{mL}^{-1}$, respectively, for quantitative analyzing. Each sample was injected in triplicate. The contents of anthraquinones were calculated according to a standard curve. Limit of determination (LOD) is the lowest concentration of an analyte that can be detected, while limit of quantitation (LOQ) is defined as the lowest concentration of a targeted analyte that can be accurately and precisely quantified. Typically, they are three and ten folds of the noise level (signal-to-noise ratio $(\mathrm{S} / \mathrm{N})=3$ and 10 ), respectively. For each targeted ingredient, both LOD and
LOQ were determined by serial dilution of stock solution under the described q-HNMR parameters (Table S1).

\section{Results and Discussion}

\section{Selecting NMR solvent}

To select an optimal solvent for NMR analysis, Rheum palmatum L. (RP) extract was applied for ${ }^{1} \mathrm{H}$ NMR experiments. The preliminary ${ }^{1} \mathrm{H}$ NMR spectra of sample dissolved in the full soluble solvents or solvent mixtures, including acetone- $d_{6}$, chloroform- $d /$ acetone- $d_{6}$ (50:50, $\mathrm{v} / \mathrm{v})$, chloroform- $d$, and DMSO- $d_{6}$, were determined and are shown in Figure 2. The solvent was chosen based on the obtained signal dispersion in the ${ }^{1} \mathrm{H}$ NMR spectral region. In this regard, acetone- $d_{6}$ and DMSO- $d_{6}$ provided the nearly satisfactory separation of key resonances. However, the ${ }^{1} \mathrm{H}$ NMR signal of the $\mathrm{OCH}_{3}$ from physcion detected in DMSO- $d_{6}$ was overlapped by signal from $\mathrm{H}_{2} \mathrm{O}$. Therefore, acetone- $d_{6}$ was selected as an optimal solvent for determination of anthraquinones in Radix et Rhizoma Rhei.

\section{Qualitative NMR analysis}

The signals of the five main anthraquinones could be observed from the ${ }^{1} \mathrm{H}$ NMR spectrum of RP according to the previous preliminary experiment and comparison with literature. ${ }^{46}$ To clarify the signal of every anthraquinone, five main bioactive anthraquinones including rhein, physcion, chrysophanol, aloe-emodin, and emodin, were dissolved in acetone- $d_{6}$ and determined by using previous ${ }^{1} \mathrm{H}$ NMR method. The results in Figures 3 and 4 show that the individual signal of every anthraquinone could be distinguished. Comparison of the ${ }^{1} \mathrm{H}$ NMR spectra of standards and samples revealed that the individual signals at $\delta_{\mathrm{H}} 8.37,6.69$, and 4.81 corresponded to rhein (H-4), emodin (H-7), and aloe-emodin (3- $\left.\mathrm{CH}_{2} \mathrm{OH}\right)$, respectively. The signals observed at $\delta_{\mathrm{H}} 6.82$ and 4.02 corresponded to the $\mathrm{H}-7$ and $6-\mathrm{OCH}_{3}$ of physcion, respectively. The signals of chrysophanol were at $\delta_{\mathrm{H}} 7.64(\mathrm{H}-4)$ and 7.20 (H-2). Inevitably, a series of additional resonances could be observed, especially in the high field region $\left(\delta_{\mathrm{H}} 0.5-2.0\right.$ and 5.32-5.38) which might be from the fatty acid or their ester in crude extracts of Radix et Rhizoma Rhei. The areas of $\delta_{\mathrm{H}}$ 7.28-7.61 and 7.78-7.90 (Figure S7) are the overlapped signals of anthraquinones. Therefore, these signals could not be selected for quantitation.

\section{Quantitative NMR analysis}

The q-HNMR requires that at least one non-overlapping 
(d)

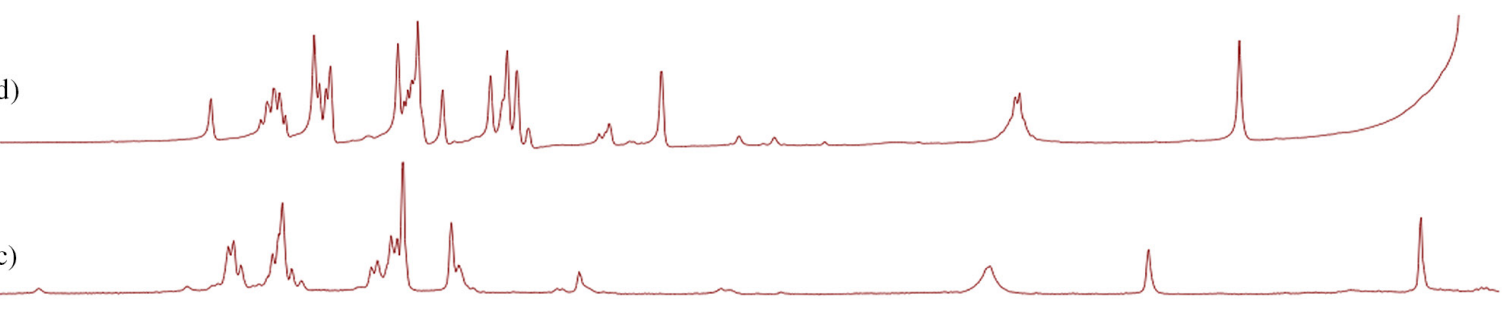

(b)

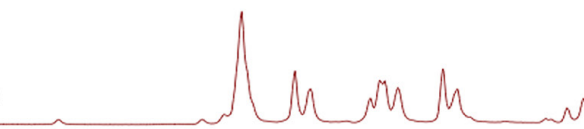

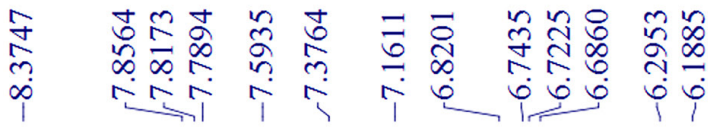

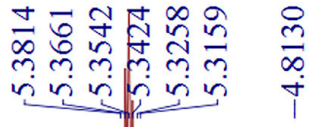

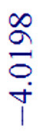

(a)
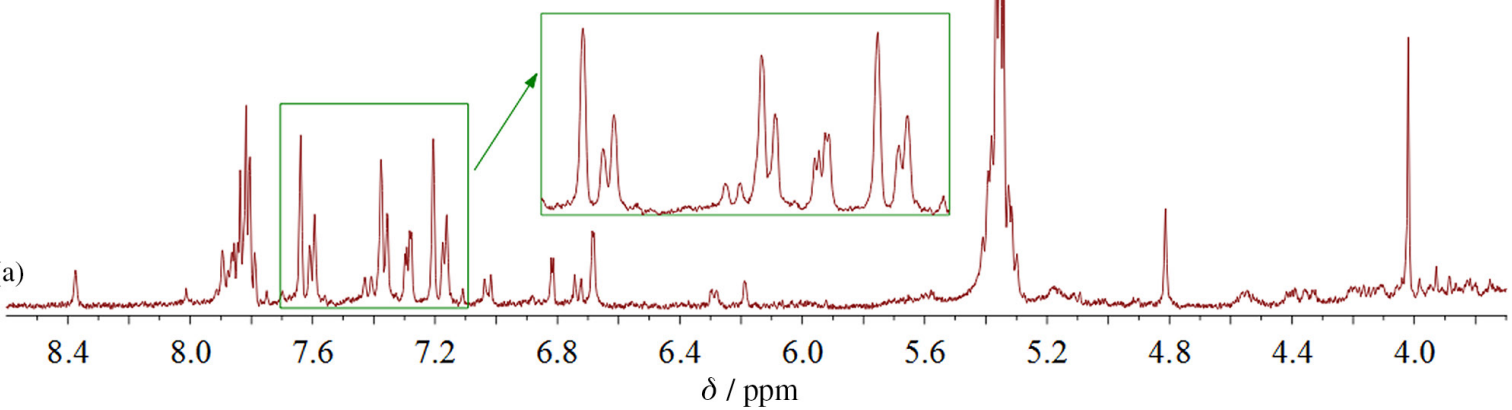

Figure 2. ${ }^{1} \mathrm{H}$ NMR spectra of $R$. palmatum extract in (a) acetone- $d_{6}$, (b) chloroform- $d /$ acetone- $d_{6}$ (50:50), (c) chloroform- $d$, and (d) DMSO- $d_{6}$.

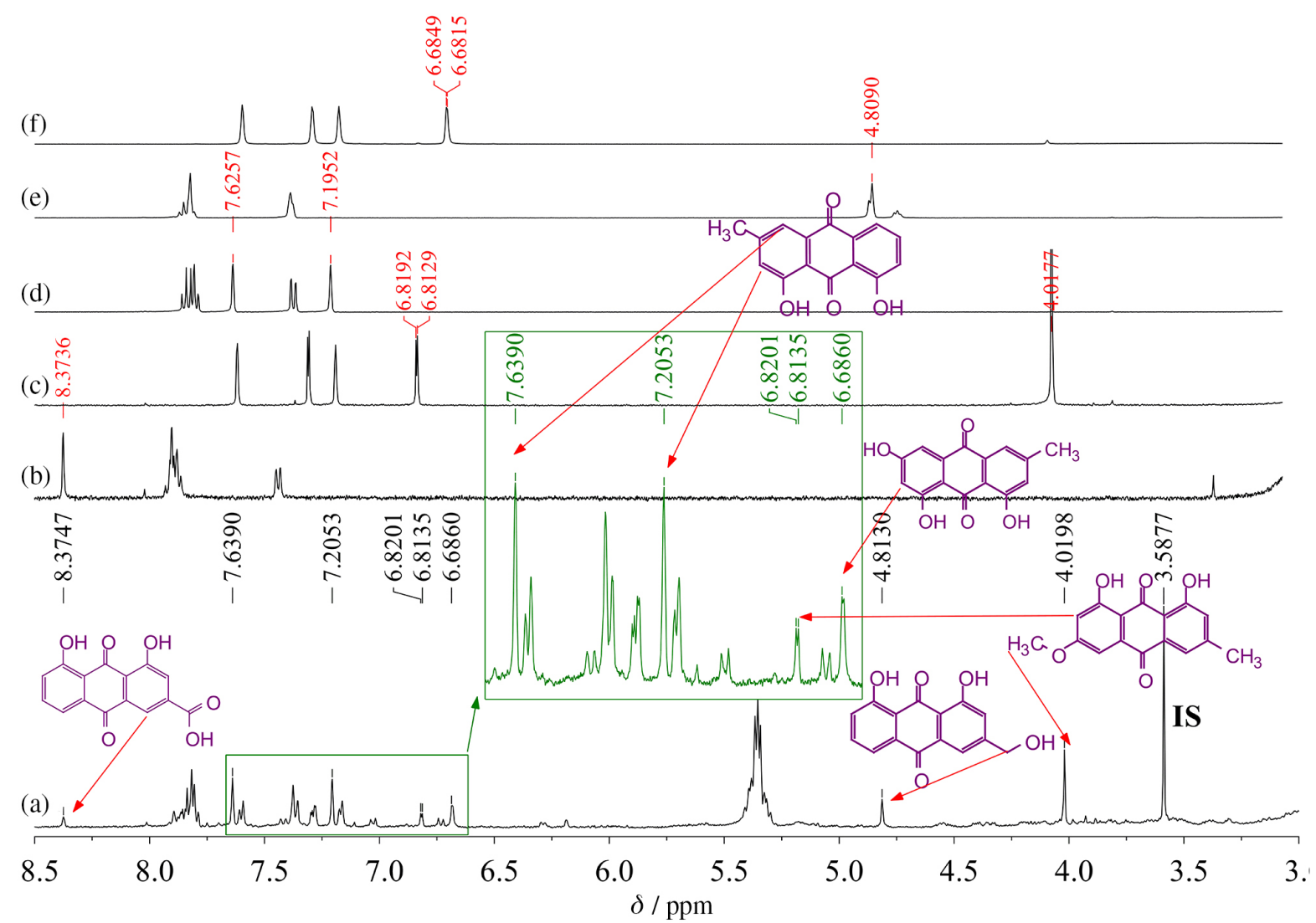

Figure 3. ${ }^{1} \mathrm{H}$ NMR spectra of (a) R. palmatum extract, (b) rhein, (c) physcion, (d) chrysophanol, (e) aloe-emodin, and (f) emodin in acetone- $d_{6}$. 


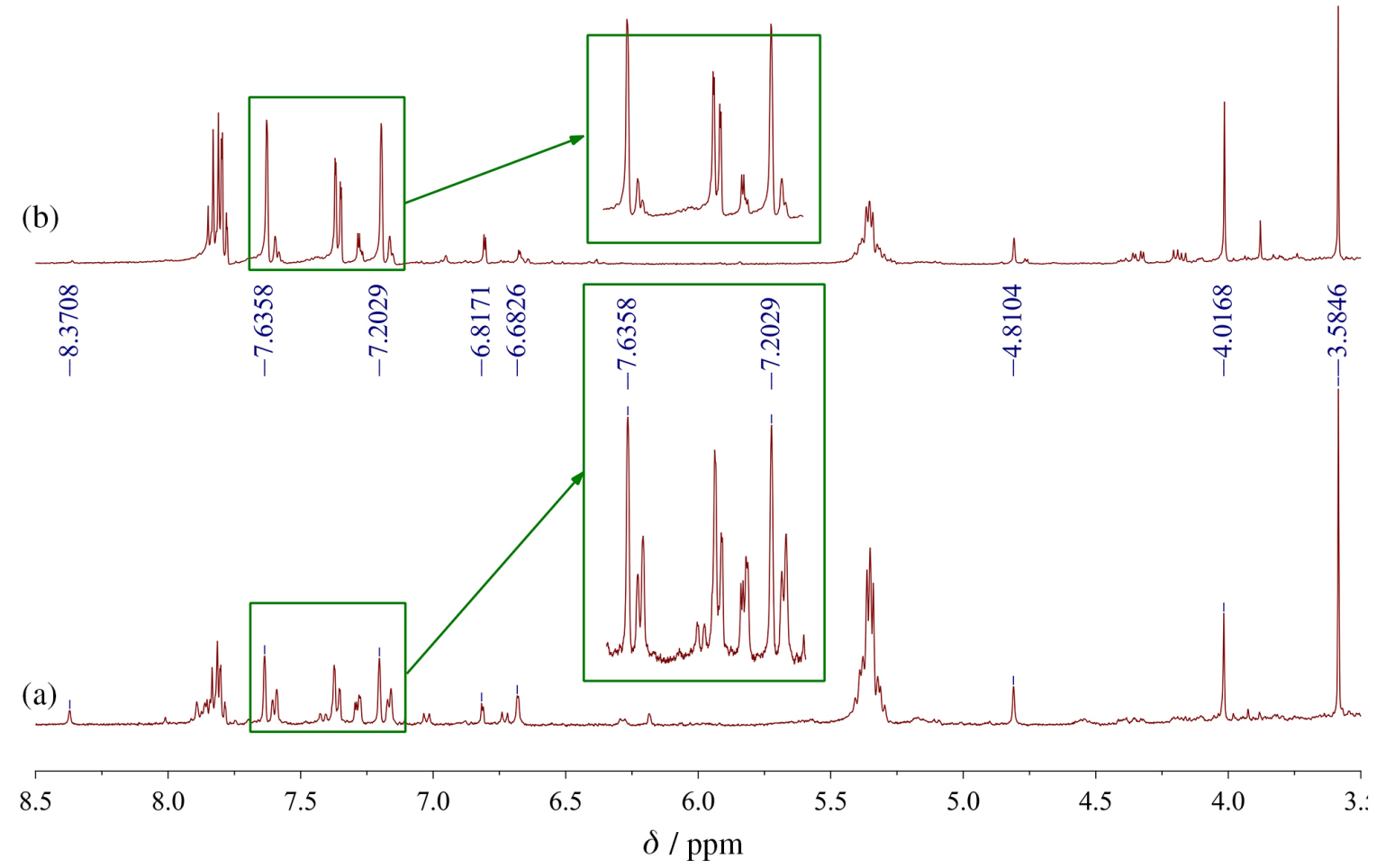

Figure 4. Detailed ${ }^{1} \mathrm{H}$ NMR spectra of (a) R. palmatum and (b) R. officinale extracts in acetone- $d_{6}$.

signal for each compound to be quantified be available for integration. ${ }^{47}$ The analysis in Qualitative NMR analysis sub-section reveals that the non-overlapping signal of five anthraquinones could be observed in the ${ }^{1} \mathrm{H}$ NMR spectrum of sample. Therefore, these five anthraquinones could be quantified by q-HNMR. Internal standard method is frequently used for quantitative NMR analysis. ${ }^{26}$ In the present study, 1,4-dioxane, piperidine, and $\mathrm{HCOONH}_{4}$ were used for selecting the internal standard ( ${ }^{1} \mathrm{H}$ NMR spectra are not shown). 1,4-Dioxane was satisfiying for q-NMR analysis of five anthraquinones in Radix et Rhizoma Rhei because its singlet at $\delta_{\mathrm{H}} 3.59(8 \mathrm{H})$ does not overlap with signals arising from constituents or solvent in the samples (Figure 3a). The contents $\left(\mathrm{mg} \mathrm{g}^{-1}\right)$ and signals chosen for quantitation of anthraquinones are shown in Table 1. The contents of physcion determined on the basis of two individual ${ }^{1} \mathrm{H}$ NMR signals at $\delta_{\mathrm{H}} 4.02$ and 6.82 were not consistent, which is discussed in Method validation sub-section.

\section{Method validation}

The q-HNMR method was validated in terms of

Table 1. Contents $\left(\mathrm{mg} \mathrm{g}^{-1}\right)$ of free anthraquinones determined by ${ }^{1} \mathrm{H}$ NMR and HPLC

\begin{tabular}{|c|c|c|c|c|c|c|c|}
\hline \multirow{2}{*}{ Method } & \multirow{2}{*}{$\begin{array}{c}\text { Rhein } \\
\delta_{\mathrm{H}} 8.37\end{array}$} & \multicolumn{2}{|c|}{ Chrysophanol } & \multirow{2}{*}{$\begin{array}{c}\text { Emodin } \\
\delta_{\mathrm{H}} 6.69\end{array}$} & \multirow{2}{*}{$\frac{\text { Aloe-emodin }}{\delta_{\mathrm{H}} 4.81}$} & \multicolumn{2}{|c|}{ Physcion } \\
\hline & & $\delta_{\mathrm{H}} 7.64$ & $\delta_{\mathrm{H}} 7.20$ & & & $\delta_{\mathrm{H}} 4.02$ & $\delta_{\mathrm{H}} 6.82$ \\
\hline \multicolumn{8}{|c|}{ Rheum palmatum $\mathrm{L}$. } \\
\hline${ }^{1} \mathrm{H} \mathrm{NMR}$ & $0.354(1.14)^{\mathrm{a}}$ & $1.350(1.10)$ & $1.343(1.83)$ & $0.907(0.31)$ & $0.410(0.71)$ & $0.644(0.09)$ & $0.522(0.24)$ \\
\hline HPLC & $0.343(3.14)$ & $1.300(1.28)$ & - & $0.935(2.72)$ & $0.408(1.22)$ & $0.537(4.67)$ & - \\
\hline $\mathrm{RSD}^{\mathrm{b}} / \%$ & 3.27 & 3.77 & 3.25 & 3.08 & 0.45 & 18.06 & 2.83 \\
\hline \multicolumn{8}{|c|}{ Rheum officinale Baill } \\
\hline${ }^{1} \mathrm{H}$ NMR & $0.078(28.21)$ & $4.958(2.26)$ & $4.906(1.62)$ & $0.515(1.79)$ & $0.353(1.02)$ & $1.126(0.73)$ & $0.996(2.44)$ \\
\hline HPLC & $0.077(2.62)$ & $4.990(0.65)$ & - & $0.506(0.42)$ & $0.355(1.28)$ & $1.032(2.41)$ & - \\
\hline $\mathrm{RSD}^{\mathrm{b}} / \%$ & 0.77 & 0.64 & 1.70 & 1.67 & 1.37 & 8.66 & 3.55 \\
\hline
\end{tabular}

${ }^{\mathrm{a}}$ The values in parentheses are presented as RSD (\%); brelative standard deviation between the results from q-HNMR and HPLC. $\delta_{\mathrm{H}}$ : hydrogen chemical shift. 
precision, stability, repeatability, and accuracy. The precision of q-HNMR analysis in Table 1 indicates that all anthraquinones could be quantified with a good precision except rhein in RO, which is attributed to its low content, implying that q-HNMR method might be not suitable for quantitation of trace constituents. ${ }^{26}$ For stability and repeatability, the ${ }^{1} \mathrm{H}$ NMR spectra of all samples were determined during 0-5 days. The results shown in Table 2 suggest that determination and quantitation of all samples could be measured during 0-5 days, except rhein in RO which might be attributed to its low content. HPLC method for determination and quantitation of anthraquinones was reliable according to the literature. ${ }^{19}$ The comparison of the results between HPLC and q-HNMR shown in Table 1 indicates that rhein, emodin, and aloe-emodin could be quantified accurately by using the individual signals from ${ }^{1} \mathrm{H}$ NMR spectroscopy. Chrysophanol could be quantified accurately using either one of two signals at $\delta_{\mathrm{H}} 7.64$ and 7.20 because of the good precision and accuracy in the quantitation by using both signals. For physcion, the signal at $\delta_{\mathrm{H}} 6.82$ could be selected for quantitation accurately, while the signal at $\delta_{\mathrm{H}} 4.02$ was unavailable because of its poor accuracy. We guess there might be some undesirable signal overlapped at $\delta_{\mathrm{H}} 4.02$, resulting in the higher integral of this peak. Accordingly, the content of physcion obtained by the signal at $\delta_{\mathrm{H}} 4.02$ increased. Thus, the ${ }^{1} \mathrm{H}$ NMR method for quantitation of five free anthraquinones in Radix et Rhizoma Rhei was established. Comparing with the quantitation by HPLC, q-HNMR method is a rapid, accuracy and stable method, and especially, it does not depend on the standard compounds for analyte identification or quantification.

\section{Conclusions}

This study presents q-HNMR used as a fast method for the determination and quantitation of five bioactive free anthraquinones in Radix et Rhizoma Rhei. The results showed that the solvent acetone- $d_{6}$ enabled satisfactory separation of the signals to be integrated in ${ }^{1} \mathrm{H}$ NMR spectrum. Five anthraquinones in Radix et Rhizoma Rhei could be quantified accurately using featured signals from ${ }^{1} \mathrm{H}$ NMR. This work implied that q-HNMR represents a feasible alternative to HPLC-based methods for quantitation of anthraquinones in Radix et Rhizoma Rhei and is suitable for the quality control of Radix et Rhizoma Rhei.

\section{Supplementary Information}

Supplementary information (data of LODs and LOQs for q-HNMR, regression equation and linear range for HPLC, HPLC chromatograms, and ${ }^{1} \mathrm{H}$ NMR spectra) is available free of charge at http://jbcs.sbq.org.br as PDF file.

Table 2. Stability of quantitative method for five free anthraquinones by ${ }^{1} \mathrm{H}$ NMR

\begin{tabular}{|c|c|c|c|c|c|c|c|}
\hline \multirow{2}{*}{ time / day } & \multirow{2}{*}{$\begin{array}{c}\text { Rhein } \\
\delta_{\mathrm{H}} 8.37\end{array}$} & \multicolumn{2}{|c|}{ Chrysophanol } & \multirow{2}{*}{$\begin{array}{c}\text { Emodin } \\
\delta_{\mathrm{H}} 6.69\end{array}$} & \multirow{2}{*}{$\frac{\text { Aloe-emodin }}{\delta_{\mathrm{H}} 4.81}$} & \multicolumn{2}{|c|}{ Physcion } \\
\hline & & $\delta_{\mathrm{H}} 7.64$ & $\delta_{\mathrm{H}} 7.20$ & & & $\delta_{\mathrm{H}} 8.37$ & $\delta_{\mathrm{H}} 7.64$ \\
\hline \multicolumn{8}{|c|}{ Rheum palmatum $\mathrm{L}$. } \\
\hline 0 & 0.354 & 1.350 & 1.343 & 0.907 & 0.410 & 0.644 & 0.522 \\
\hline 1 & 0.362 & 1.360 & 1.341 & 0.903 & 0.413 & 0.646 & 0.517 \\
\hline 2 & 0.360 & 1.367 & 1.357 & 0.905 & 0.408 & 0.646 & 0.524 \\
\hline 3 & 0.358 & 1.352 & 1.348 & 0.898 & 0.410 & 0.645 & 0.520 \\
\hline 4 & 0.367 & 1.381 & 1.350 & 0.898 & 0.408 & 0.651 & 0.520 \\
\hline 5 & 0.364 & 1.366 & 1.341 & 0.906 & 0.406 & 0.645 & 0.523 \\
\hline $\mathrm{RSD}^{\mathrm{a}} / \%$ & 1.28 & 0.83 & 0.47 & 0.44 & 0.60 & 0.38 & 0.47 \\
\hline \multicolumn{8}{|c|}{ Rheum officinale Baill } \\
\hline 0 & 0.078 & 4.958 & 4.906 & 0.515 & 0.353 & 1.126 & 0.996 \\
\hline 1 & 0.097 & 5.035 & 4.915 & 0.512 & 0.358 & 1.126 & 0.998 \\
\hline 2 & 0.079 & 5.015 & 4.956 & 0.515 & 0.350 & 1.123 & 1.000 \\
\hline 3 & 0.076 & 4.980 & 4.924 & 0.510 & 0.351 & 1.126 & 1.000 \\
\hline 4 & 0.080 & 4.934 & 4.921 & 0.516 & 0.349 & 1.126 & 0.989 \\
\hline 5 & 0.077 & 4.999 & 4.938 & 0.524 & 0.355 & 1.128 & 0.992 \\
\hline $\mathrm{RSD}^{\mathrm{a}} / \%$ & 9.78 & 0.74 & 0.36 & 0.91 & 0.98 & 0.15 & 0.45 \\
\hline
\end{tabular}

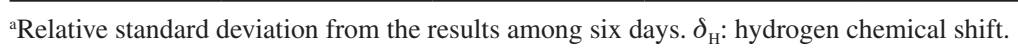




\section{Acknowledgments}

This work was financially supported with two grants from the Natural Science Foundation of China (No. 81460648 and 81460536), a grant from the Program for Changjiang Scholars and Innovative Research Team in University (IRT13095), and a grant from New Academic Researcher Award for Doctoral Candidates of Yunnan Province.

\section{References}

1. Chinese Pharmacopoeia Committee; Chinese Pharmacopoeia, vol. 1; China Medical Science Press: Beijing, 2010.

2. Hu, S. S.; Cao, W.; Dai, H. B.; Da, J. H.; Ye, L. H.; Cao, J.; Li, X. Y.; J. Agric. Food. Chem. 2014, 62, 8822.

3. Wang, G. Y.; Shi, Y. P.; Acta Chromatogr. 2014, 26, 229.

4. Yang, F.; Xu, Y.; Xiong, A.; He, Y.; Yang, L.; Wan, Y.-J. Y.; Wang, Z.; J. Ethnopharmacol. 2012, 144, 599.

5. Fu, X. S.; Chen, F.; Liu, X. H.; Xu, H.; Zhou, Y. Z.; Chin. J. New Drugs 2011, 20, 1534.

6. Wang, G. Y.; Qi, H. Y.; Shi, Y. P.; J. Sep. Sci. 2010, 33, 1730.

7. Xiao, P.; He, L.; Wang, L.; J. Ethnopharmacol. 1984, 10, 275.

8. Wang, G. Y.; Chen, F. F.; Shi, Y. P.; J. Sep. Sci. 2011, 34, 268.

9. Zhu, J. J.; Wang, Z. M.; Ma, X. Y.; Feng, W.-H.; Zhang, Q. Y.; Chin. Herb. Med. 2012, 4, 157.

10. Zhang, W.; Sun, J.; Wang, Y.; Liu, X.; Sun, Y.; Lu, R.; He, Z.; Chromatographia 2009, 70, 1321.

11. Lin, C.-C.; Wu, C.-I.; Lin, T.-C.; Sheu, S.-J.; J. Sep. Sci. 2006, 29, 2584.

12. Ohshima, Y.; Takahashi, K.; J. Chromatogr. A 1983, 258, 292.

13. Kashiwada, Y.; Nonaka, G.; Nishioka, I.; Chem. Pharm. Bull. 1989, 37, 999.

14. Poulsen, J. R.; Birks, J. W.; Anal. Chem. 1989, 61, 2267.

15. Djozan, D.; Assadi, Y.; Talanta 1995, 42, 861.

16. Liu, C. L.; Zhu, P. L.; Liu, M. C.; J. Chromatogr. A 1999, 857, 167.

17. Ohshima, Y.; Ohno, Y.; Kajiyama, K.; Takahashi, K.; J. Chromatogr. A 1986, 360, 303.

18. Zhang, H. F.; Shi, Y. P.; Talanta 2010, 82, 1010.

19. Zhang, C.; Li, L.; Xiao, Y. Q.; Lin, N.; Liu, C. F.; Li, G. L.; Pang, Z.; Chen, D. D.; Tian, G. F.; China J. Chin. Mater. Med. 2009, 34, 1914.

20. Ma, X.; Chen, Y.; Hui, R.; Chromatographia 1989, 27, 465.

21. Nyiredy, S.; Dallenbach-Toelke, K.; Zogg, G. C.; Sticher, O.; J. Chromatogr. A 1990, 499, 453.

22. Nonaka, G.; Nishioka, I.; Nagasawa, T.; Oura, H.; Chem. Pharm. Bull. 1981, 29, 2862.

23. Kashiwada, Y.; Nonaka, G.-I.; Nishioka, I.; Chem. Pharm. Bull. 1986, 34, 4083 .
24. Wang, J.; Li, H.; Jin, C.; Qu, Y.; Xiao, X.; J. Pharm. Biomed. Anal. 2008, 47, 765.

25. Yang, F.; Zhang, T.; Tian, G.; Cao, H.; Liu, Q.; Ito, Y.; J. Chromatogr. A 1999, 858, 103.

26. Pieri, V.; Belancic, A.; Morales, S.; Stuppner, H.; J. Agric. Food. Chem. 2011, 49, 4378.

27. Pauli, G. F.; Godecke, T.; Jaki, B. U.; Lankin, D. C.; J. Nat. Prod. 2012, 75, 834 .

28. Huang, F.; Pan, S.; Pu, Y.; Ben, H.; Ragauskas, A. J.; RSC Adv. 2014, 4, 17743.

29. Frank, O.; Kreissl, J. K.; Daschner, A.; Hofmann, T.; J. Agric. Food. Chem. 2014, 62, 2506.

30. Muller-Maatsch, J.; Caligiani, A.; Tedeschi, T.; Elst, K.; Sforza, S.; J. Agric. Food. Chem. 2014, 62, 9081.

31. Yuan, Y.; Song, Y.; Jing, W.; Wang, Y.; Yang, X.; Liu, D.; Anal. Methods 2014, 6, 907.

32. Bussy, U.; Giraudeau, P.; Tea, I.; Boujtita, M.; Talanta 2013, 116, 554.

33. Lee, S. G.; Hyun, S.-H.; Sung, G.-H.; Choi, H.-K.; Anal. Lett. 2014, 47, 1031.

34. de Graaf, R. A.; Chowdhury, G. M.; Behar, K. L.; Anal. Chem. 2014, 86, 5032.

35. Schicho, R.; Nazyrova, A.; Shaykhutdinov, R.; Duggan, G.; Vogel, H. J.; Storr, M.; J. Proteome Res. 2010, 9, 6265.

36. Gowda, G. A. N.; Tayyari, F.; Ye, T.; Suryani, Y.; Wei, S.; Shanaiah, N.; Raftery, D.; Anal. Chem. 2010, 82, 8983.

37. de Graaf, R. A.; Behar, K. L.; Anal. Chem. 2003, 75, 2100.

38. Ohtsuki, T.; Sato, K.; Abe, Y.; Sugimoto, N.; Akiyama, H.; Talanta 2015, 131, 712.

39. del Campo, G.; Berregi, I.; Caracena, R.; Zuriarrain, J.; Talanta 2010, $81,367$.

40. Neumüller, K. G.; Carvalho de Souza, A.; Van Rijn, J.; Appeldoorn, M. M.; Streekstra, H.; Schols, H. A.; Gruppen, H.; J. Agric. Food. Chem. 2013, 61, 6282.

41. Valverde, J.; This, H.; J. Agric. Food. Chem. 2007, 56, 314.

42. Branch, S. K.; J. Pharm. Biomed. Anal. 2005, 38, 798.

43. Malz, F.; Jancke, H.; J. Pharm. Biomed. Anal. 2005, 38, 813.

44. Sharma, R.; Gupta, P. K.; Mazumder, A.; Dubey, D. K.; Ganesan, K.; Vijayaraghavan, R.; J. Pharm. Biomed. Anal. 2009, 49, 1092.

45. Webster, G.; Kumar, S.; Anal. Chem. 2014, 86, 11474.

46. Cui, X. R.; Tsukada, M.; Suzuki, N.; Shimamura, T.; Gao, L.; Koyanagi, J.; Komada, F.; Saito, S.; Eur. J. Med. Chem. 2008, 43, 1206.

47. Beyer, T.; Schollmayer, C.; Holzgrabe, U.; J. Pharm. Biomed. Anal. 2010, 52, 51.

Submitted: March 2, 2016

Published online: April 6, 2016 